\title{
42 ASSESSING FATHERS' ATTITUDES TOWARDS PROTECTING CHILDREN FROM INJURIES AND ENGAGING IN PHYSICAL RISKS
}

doi:10.1136/injuryprev-2012-040590d.42

Olsen Lise, Brussoni Mariana, Ishikawa Takuro, Mâsse Louise. University of British Columbia, 4480 Oak Street, Vancouver, British Columbia, Canada

Background While most child injury prevention research has focused on mothers, research suggests fathers also play an important role. In a recent study, fathers described how they constantly balanced efforts to expose their children to new and potentially risky situations with needs to protect children from injury.

Aim To develop and validate an instrument that measures fathers' attitudes and practices towards promoting risk engagement for their children and towards protective strategies to reduce injury risk.

Methods We developed a survey instrument for testing with 300 fathers of children ages 6-12 years accessing services at a Canadian paediatric hospital. Survey items were developed using findings from past qualitative research with fathers, and existing literature. Content validity assessment was completed using expert opinion and cognitive interviews with fathers. The full sample consists of fathers with children attending hospital due to injury as well as non-injury reasons.

Results Survey development included opinion from three content experts and cognitive interviews with five fathers. Items include fathers' attitudes and practices related to protecting children from injuries and children's engagement with risky, physical activities. Questions also address child injury history and fathers' perceptions of their role and level of involvement. Results will be compared and reported for the injured and the non-injured groups.

Contribution Understanding fathers' attitudes towards child risk and safety is important for designing prevention messages that fit with their views on parenting. Potential uses for the instrument for further research and injury prevention practice will be discussed. 